\title{
Théâtres de Pompéi
}

Campagnes 2017

Éloïse Letellier-Taillefer et Guilhem Chapelin

\section{(2) OpenEdition \\ 1 Journals}

Édition électronique

URL : http://journals.openedition.org/cefr/3246

ISSN : 2282-5703

\section{Éditeur}

École française de Rome

\section{Référence électronique}

Éloïse Letellier-Taillefer et Guilhem Chapelin, «Théâtres de Pompéi », Chronique des activités archéologiques de l'École française de Rome [En ligne], Les cités vésuviennes, mis en ligne le 23 septembre 2019, consulté le 23 septembre 2019. URL : http://journals.openedition.org/cefr/3246

\footnotetext{
Ce document a été généré automatiquement le 23 septembre 2019

(c) École française de Rome
} 


\title{
Théâtres de Pompéi
}

\author{
Campagnes 2017
}

\section{Éloïse Letellier-Taillefer et Guilhem Chapelin}

\section{NOTE DE L'ÉDITEUR}

Éloïse Letellier-Taillefer (EFR) assure la coordination scientifique du projet. Guilhem Chapelin, architecte (CJB) a participé à l'étude des blocs de la summa cavea et au relevé photogrammétrique des parois du couloir VIII 7, 20. Thomas Crognier, photographe, et Javiera Hiault-Echeverria, dessinatrice, ont participé au relevé graphique et photogrammétrique et à l'étude des graffitis du couloir VIII 7, 20 .

\section{NOTE DE L'AUTEUR}

Nous tenons à remercier les institutions qui rendent possibles les recherches menées dans le cadre du projet Teatri di Pompei. Nous adressons en particulier nos plus vifs remerciements aux équipes du Parco Archeologico di Pompei : au directeur général Massimo Osanna, à Grete Stefani, directrice des fouilles et à Laura D'Esposito, alors fonctionnaire responsable de la regio VIII, pour le soutien accordé à ce projet de recherche et le support fourni pendant les campagnes de terrain.

\section{Introduction}

1 En 2017 le projet Teatri di Pompei ${ }^{1}$ a poursuivi ses travaux de terrain dans le cadre de deux brèves campagnes consacrées à des enquêtes thématiques ciblées. La première, conduite sur quelques jours entre février et mars 2017, a concerné l'analyse de la summa cavea du grand théâtre et du dispositif de fixation du velum. La seconde, du 12 au 16 juillet 2017, a permis d'entreprendre le relevé graphique et photographique des graffitis antiques qui couvrent les murs du couloir d'accès aux deux théâtres (VIII 7, 
20). Nous exposons ici les résultats préliminaires de ces travaux, dont l'analyse se poursuit.

\section{Étude du dispositif de fixation du velum dans le grand théâtre}

2 L'enquête sur le dispositif de fixation du velum dans le grand théâtre de Pompéi illustre la situation documentaire complexe de cet édifice. Retrouvé dans un état de conservation exceptionnel sous les matériaux éruptifs du Vésuve à la fin du xvIII siècle, il a fait l'objet de restaurations précoces et conséquentes. Celles-ci sont partiellement documentées par les archives et les témoignages des visiteurs du site mais demeurent très mal connues scientifiquement ${ }^{2}$, alors que l'édifice joue un rôle clé dans le développement de l'architecture théâtrale antique.

3 Avec la summa cavea et le dispositif de fixation du velum (consoles percées et corniche sommitale avec encastrements pour les mâts), ces restaurations ont touché des vestiges cruciaux pour l'étude des édifices de spectacles antiques, tant par leur conservation exceptionnelle que par leur date précoce et, pour certaines caractéristiques, par leur unicité $e^{3}$ Alors que dans presque tous les édifices de spectacles les consoles soutenant les mâts sont placées à l'extérieur des murs périphériques ${ }^{4}$, celles de Pompéi sont fixées à l'intérieur. Mais peut-on se fier à cet emplacement actuel, s'il est susceptible d'être issu d'une restauration moderne ? C'est pour répondre à cette question que nous avons croisé une enquête sur la documentation moderne, écrite et graphique, avec l'étude du bâti conservé.

\section{Les restaurations de la summa cavea}

4 Comme l'indiquent les journaux de fouilles, les structures de la summa cavea du grand théâtre, construite lors de l'agrandissement augustéen du bâtiment, ont été retrouvées largement effondrées au moment de leur découverte, même s'il est encore difficile, dans l'état actuel des connaissances, de déterminer la part des bouleversements respectivement dus au séisme de 62-63 et à l'éruption de 79 apr. J.-C. dans cet état de dégradation. Nos recherches sur la documentation moderne indiquent que la crypta, les gradins de la summa cavea et le mur périphérique de cette dernière, qui sert de support aux consoles du velum dans l'état actuel, ont été largement remontés lors d'importantes restaurations menées entre 1793 et 1797. Un tableau de J.-P. Hackert illustre une première phase de ces travaux, peu avant leur achèvement à la toute fin du XvIII siècle (fig. 1, à comparer avec l'état achevé : fig. 2). Par la suite, hormis l'installation d'un mât en bois dans la troisième console, à titre de démonstration (fig. 2), l'état de cette partie du bâtiment n'évolua pas significativement. La summa cavea fut fortement endommagée par le bombardement de septembre 19435. Les structures furent progressivement restaurées dans leur état préalable, entre $1945^{6}$ et les années $1950^{7}$, avec la remise en place de certains blocs de la corniche et d'une des consoles du velum qui avait été abattue (fig. 3). Au cours des travaux de reconstruction menés dans le théâtre en 2010, les gradins de la summa cavea, comme tous les autres, ont été entièrement reconstruits en tuf, à l'exception d'une partie située à l'extrémité sud de la summa cavea, entre la porte d'accès et l'angle du bâtiment. Des barrières métalliques 
ont été installées et des mâts en bois sont aujourd'hui placés dans chacune des consoles remontées au XVIII ${ }^{\mathrm{e}}$ siècle (fig. 4).

Fig. 1 - Détail d'un tableau de J.-P. Hackert peint en 1799 et montrant l'état des restaurations de la summa cavea à une date indéterminée entre 1795 et 1797.

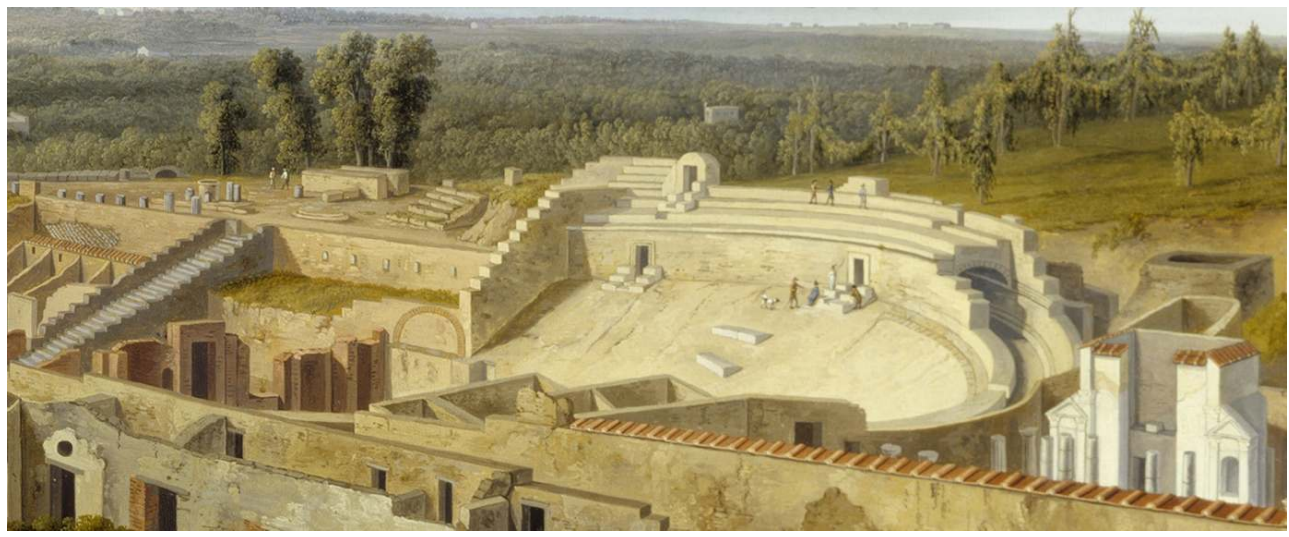

Attingham Park, Shropshire. National Trust inv. Nr. 608992.

Fig. 2 - Détail d'un panorama sur le quartier des théâtres dessiné par W. Gell avant 1819.

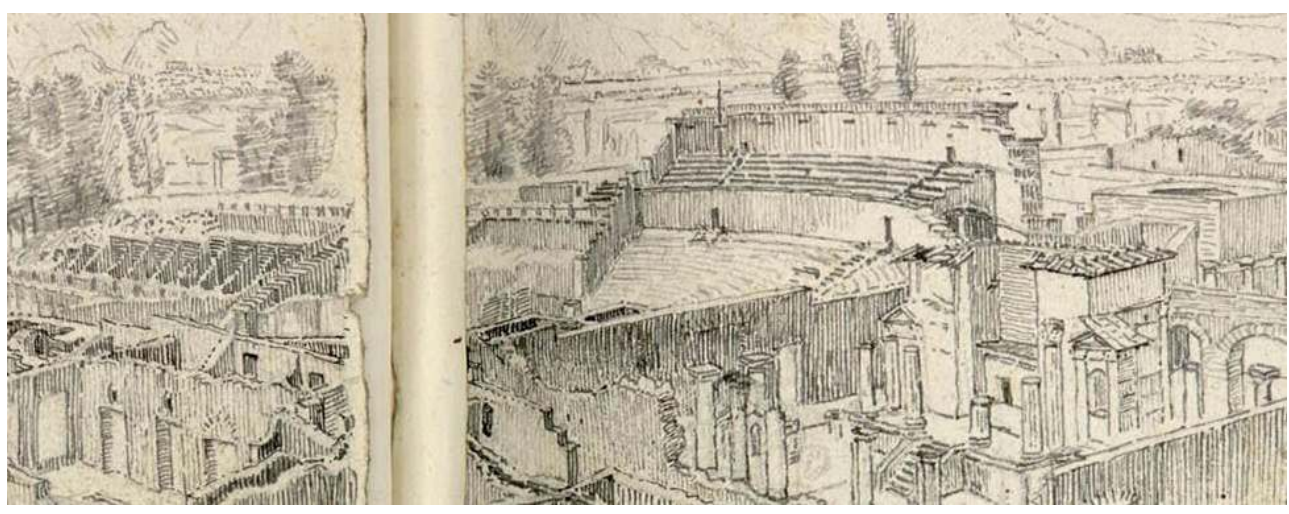

Bibliothèque de l'Institut National d'Histoire de l'Art, collections Jacques Doucet, MS 180 (1), p. 40.

Fig. 3 - Vue similaire photographiée en avril 2009.

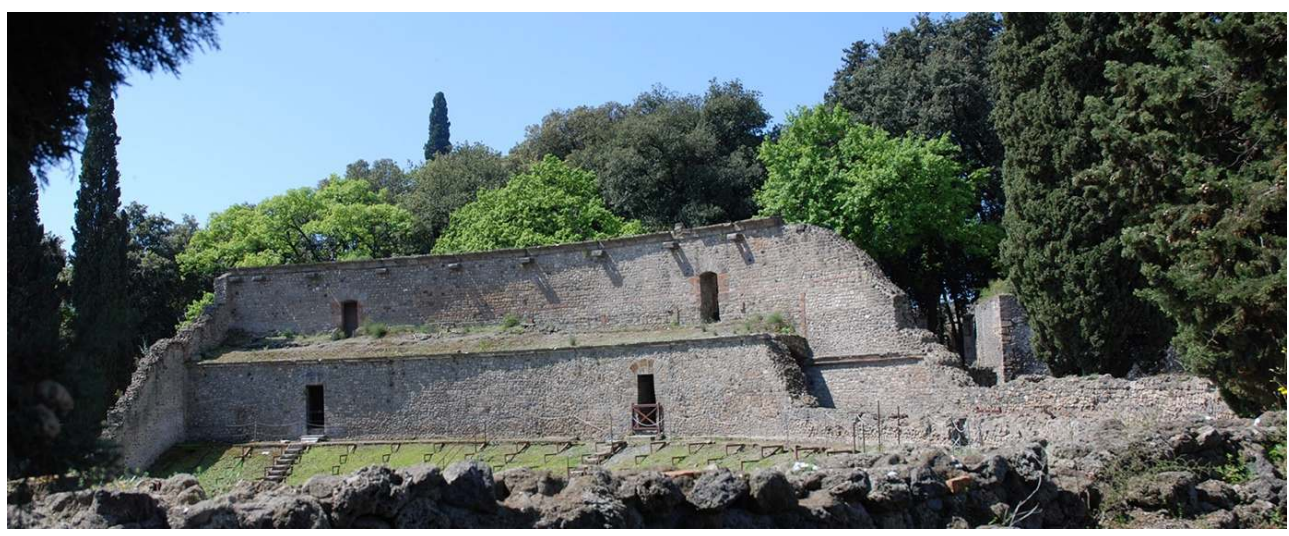

Cl. É. Letellier-Taillefer, avec l'autorisation du Parco Archeologico di Pompei, reproduction interdite. 
Fig. 4 - État actuel de la cavea du grand théâtre, après les travaux de 2010.

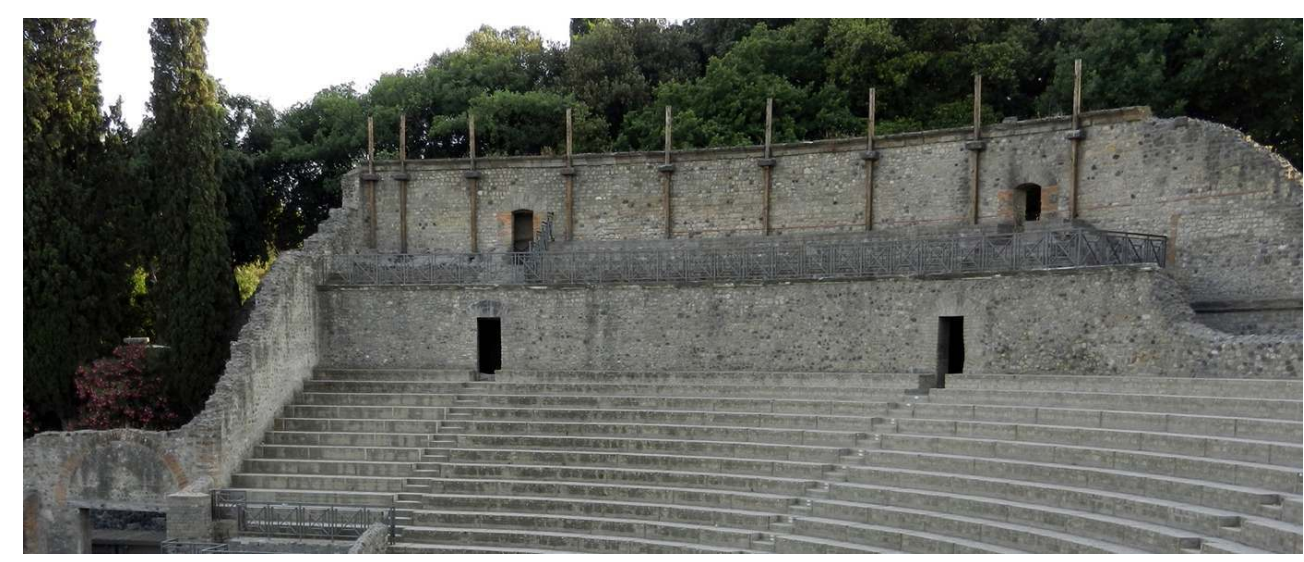

Cl. É. Letellier-Taillefer, avec l'autorisation du Parco Archeologico di Pompei, reproduction interdite.

5 Pour analyser le dispositif du velum, dans quelle mesure peut-on se fier aux restaurations dont les partis-pris remontent au xvIII ${ }^{\mathrm{e}}$ siècle; faut-il les considérer comme un reflet fidèle de la situation antique ${ }^{8}$ ? Étant donné la prudence qui caractérisait les projets et méthodes de restauration de Francesco La Vega, on peut supposer que le remontage de ces parties hautes s'appuya sur des indices précis9. Malheureusement, aucun détail n'est donné dans les rapports de fouilles sur la nature de ces indices ni sur l'état de conservation des éléments trouvés en place. On ne sait pas, par exemple, ni quand ni où furent retrouvées les consoles percées du velum ou les blocs de la corniche en tuf qui couronne aujourd'hui le mur périphérique.

6 On peut en premier lieu, en restituant l'état des connaissances sur les vela à la fin du $\mathrm{XVIII}^{\mathrm{e}}$ siècle, se demander sur quels éléments extrinsèques Francesco La Vega aurait pu s'appuyer pour proposer la restauration bâtie à Pompéi. Les principales sources à cette époque étaient textuelles : on pouvait se référer notamment à Vitruve, qui soulignait dans son chapitre sur les machines l'importance des dispositifs mécaniques associés aux spectacles, en particulier ceux destinés à tendre les vela ${ }^{10}$. Dans la littérature antérieure ou contemporaine à Francesco La Vega sur l'architecture théâtrale antique, on ne trouve pas de description précise du dispositif de déploiement du velum, toujours mentionné seulement à partir des diverses sources littéraires à travers lesquelles on l'appréhendait ${ }^{11}$. On connaissait certes le dispositif du Colisée, mais le plan des amphithéâtres posait des problèmes spécifiques; le théâtre d'Herculanum n'avait pas livré d'indices matériels sur la disposition du velum ${ }^{12}$. Même s'il faudrait élargir cette enquête historiographique ${ }^{13}$, il ne semble pas que Francesco La Vega ait pu s'appuyer sur beaucoup d'autres exemples : tout tend à confirmer que sa restauration se limitait à une remise en place des éléments épars trouvés lors des fouilles, dont on doit interroger la fiabilité. Il est pour cela nécessaire de compléter la documentation d'archives par l'analyse du bâti.

\section{Repérage des parties antiques conservées}

7 C'est dans l'angle sud-ouest de la cavea $^{14}$ que la conservation des vestiges antiques semble la plus étendue. Le premier plan connu du théâtre, établi par Jean-Augustin Renard en 1778 , après les premiers dégagements et avant les restaurations ${ }^{15}$, montre que cet angle du bâtiment était conservé au moment des fouilles, au moins jusqu'au 
niveau de circulation dans le forum triangulaire, où s'ouvrait la porte du petit escalier d'accès direct à la summa cavea.

8 À cet endroit, du côté intérieur de la summa cavea - grâce à l'érosion des gradins remontés au XVIII ${ }^{\mathrm{e}}$ siècle et à l'intervention limitée des restaurateurs de 2010 - on peut observer la maçonnerie antique. D'après notre analyse des techniques et matériaux employés, elle apparaît conservée jusqu'au départ du couvrement voûté d'une structure creuse sur laquelle nous revenons ci-dessous (fig. 5). Au-dessus de cette structure, l'élévation du mur périphérique de la summa cavea montre en revanche des signes caractéristiques des restaurations de la fin du XvIII siècle : hétérogénéité des matériaux, irrégularité de leur mise en œuvre et, indice particulièrement parlant d'après nos observations dans le théâtre, utilisation de blocs de calcaire du Sarno incisés de lignes verticales ou en croix, destinées à simuler des moellons de petit appareil.

Fig. 5 - État actuel des structures antiques dans l'angle sud-ouest de la summa cavea.

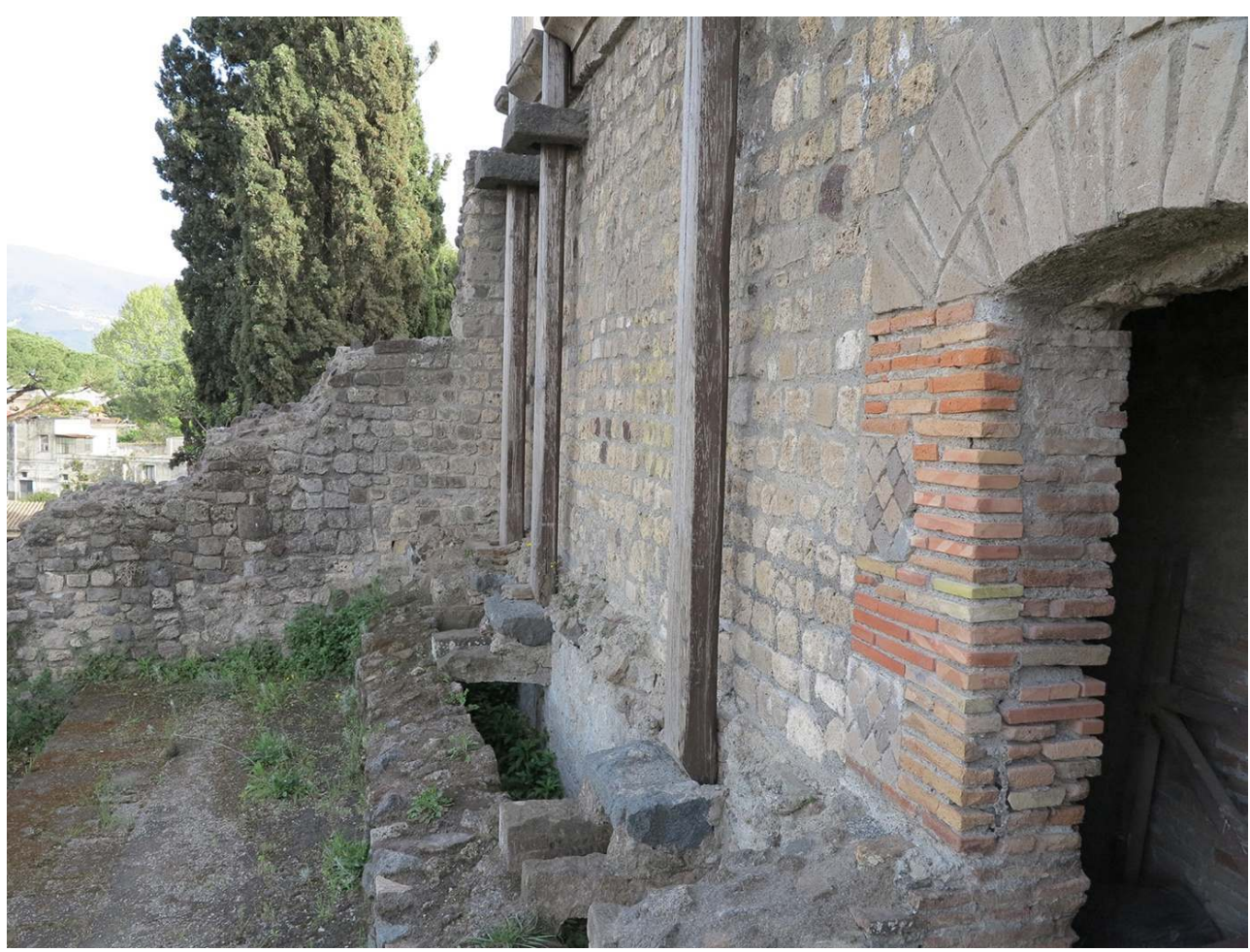

Cl. É. Letellier-Taillefer, avec l'autorisation du Parco Archeologico di Pompei, reproduction interdite.

La structure antique creuse, dont la fonction n'a encore jamais été expliquée ${ }^{16}$, ressemble à une canalisation d'environ $60 \mathrm{~cm}$ de largeur et $114 \mathrm{~cm}$ de hauteur ${ }^{17}$ délimitée à l'ouest par la partie basse du mur périphérique de la summa cavea et à l'est par un muret maçonné. Le fond de cet espace creux est aujourd'hui plan et couvert de mortier hydraulique mais R. Graefe rapporte avoir observé ce creux se prolonger jusqu'à l'extrados voûté de la crypta $^{18}$ (fig. 6). Cette structure était originellement couverte d'une voûte maçonnée qui était à son tour dissimulée sous la maçonnerie, aujourd'hui disparue, du dernier rang de gradins de la summa cavea. 
Fig. 6 - Coupes sur la crypta et la summa cavea, par R. Graefe. À gauche : au niveau d'un mât du velum ; à droite : entre deux mâts.
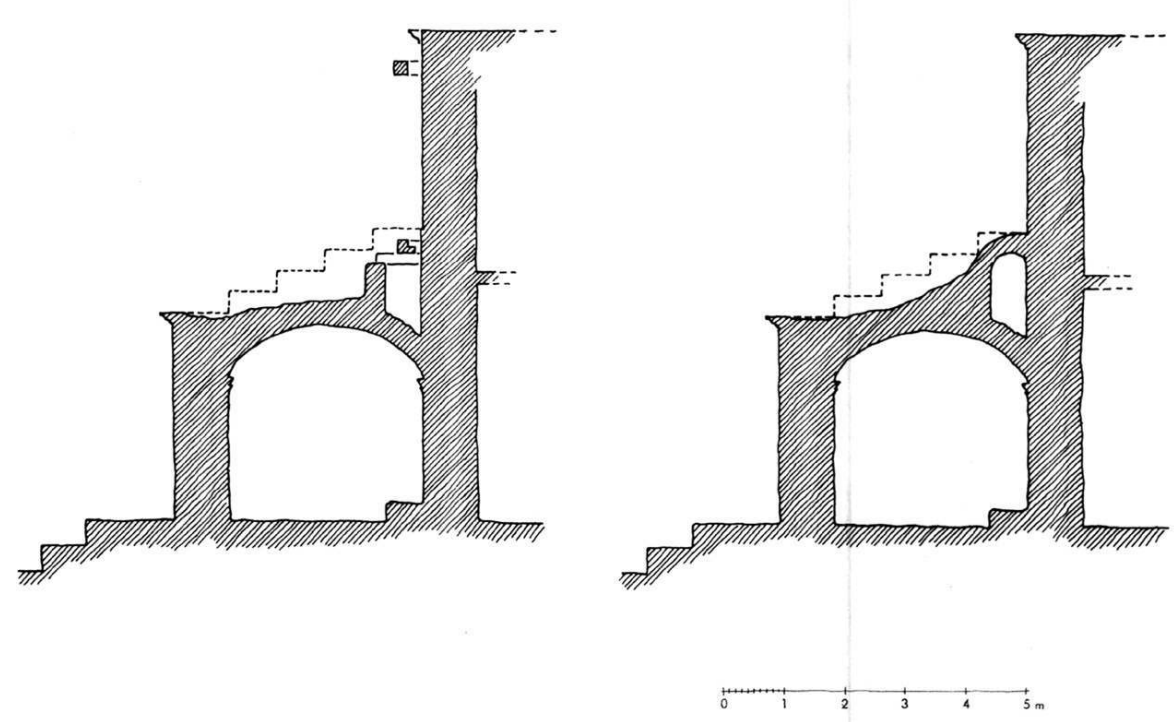

Graefe 1979, fig. 24 p. 38 intervalles irréguliers, par des cavités quadrangulaires maçonnées (fig. 7). Celles-ci étaient aménagées autour d'un noyau formé de deux blocs longs en tuf (dimensions de ceux placés sous le $2^{\mathrm{e}}$ mât : $54 \times 22 \times 18 \mathrm{~cm}$ ) reposant transversalement sur les parois de la structure creuse et soutenant un troisième bloc en lave $(60 \times 30 \times 18 \mathrm{~cm})$ disposé sur de la maçonnerie, perpendiculairement aux blocs de tuf, de manière à ménager un espace libre entre ce bloc et le mur périphérique (fig. 8).

Comme l'illustrent les mâts en bois installés en 2010 (cf. fig. 5), la restauration menée à la fin du XVIII ${ }^{e}$ siècle a consisté à placer les consoles percées du velum dans l'alignement de ces cavités maçonnées, ce qui repose sur leur interprétation comme supports inférieurs pour les mâts du velum. Dans cette hypothèse, le choix de consoles et de supports en lave s'expliquerait bien par la volonté d'utiliser pour les pièces les plus sollicitées (par les frottements liés aux mouvements des mâts pendant les manœuvres ou, lorsque le velum était en place) le matériau de construction le plus résistant à disposition. 
Fig. 7 - Structures antiques interprétées comme points d'appui des mâts du velum.

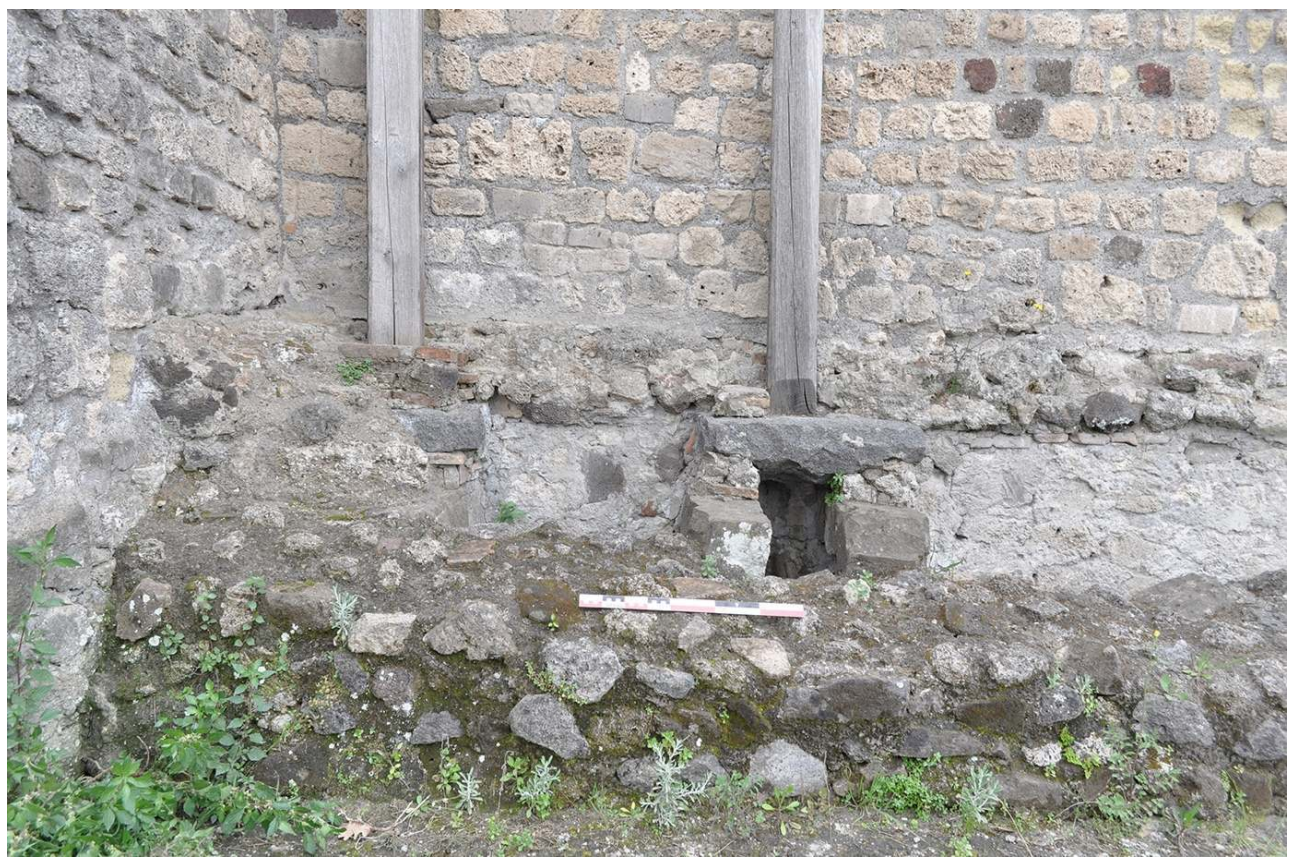

Cl. É. Letellier-Taillefer, avec l'autorisation du Parco Archeologico di Pompei, reproduction interdite.

Fig. 8 - Coupe E-O sur la summa cavea du grand théâtre de Pompéi, au niveau du $1^{\mathrm{er}}$ mât du velum.
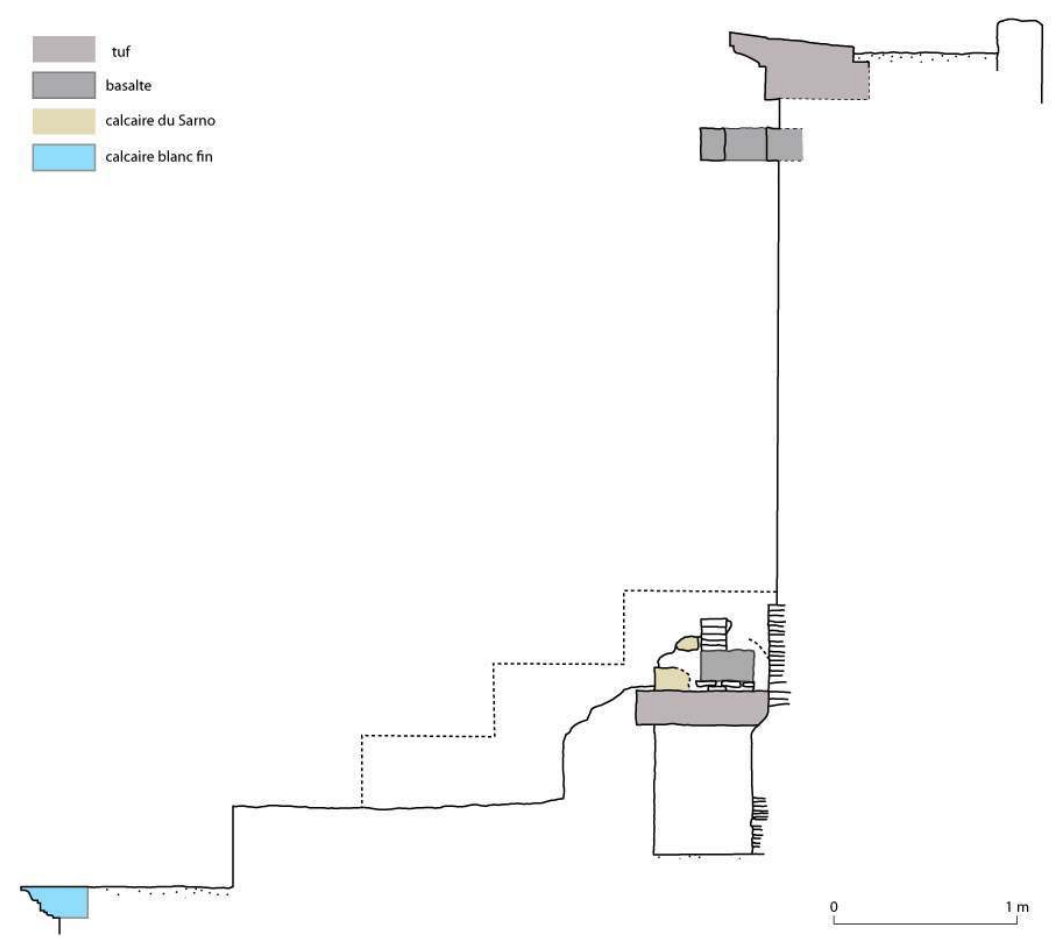

G. Chapelin, É. Letellier-Taillefer

12 Un autre indice archéologique, encore jamais relevé, à notre connaissance, vient compléter ces observations sur la conservation du bâti antique dans ce secteur. À l'extérieur du théâtre, dans l'angle formé par le mur du forum triangulaire et la façade 
sud de la cavea, on conserve une descente d'eau, constituée de tubes en terre cuite emboîtés, fixés dans l'angle du mur avec du mortier (fig. 9). Leur présence confirme la hauteur de conservation des vestiges antiques jusqu'à la structure creuse préalablement décrite. L'emploi de techniques modernes dans une très grande partie de cette façade sud ${ }^{19}$ révèle cependant que seul l'angle était préservé, tandis que le reste des élévations durent être largement remontées.

Fig. 9 - Angle sud-ouest de la cavea vu depuis le sud, au niveau de circulation de la terrasse intermédiaire entre théâtres et forum triangulaire, avec une vue de détail de la descente d'eau.

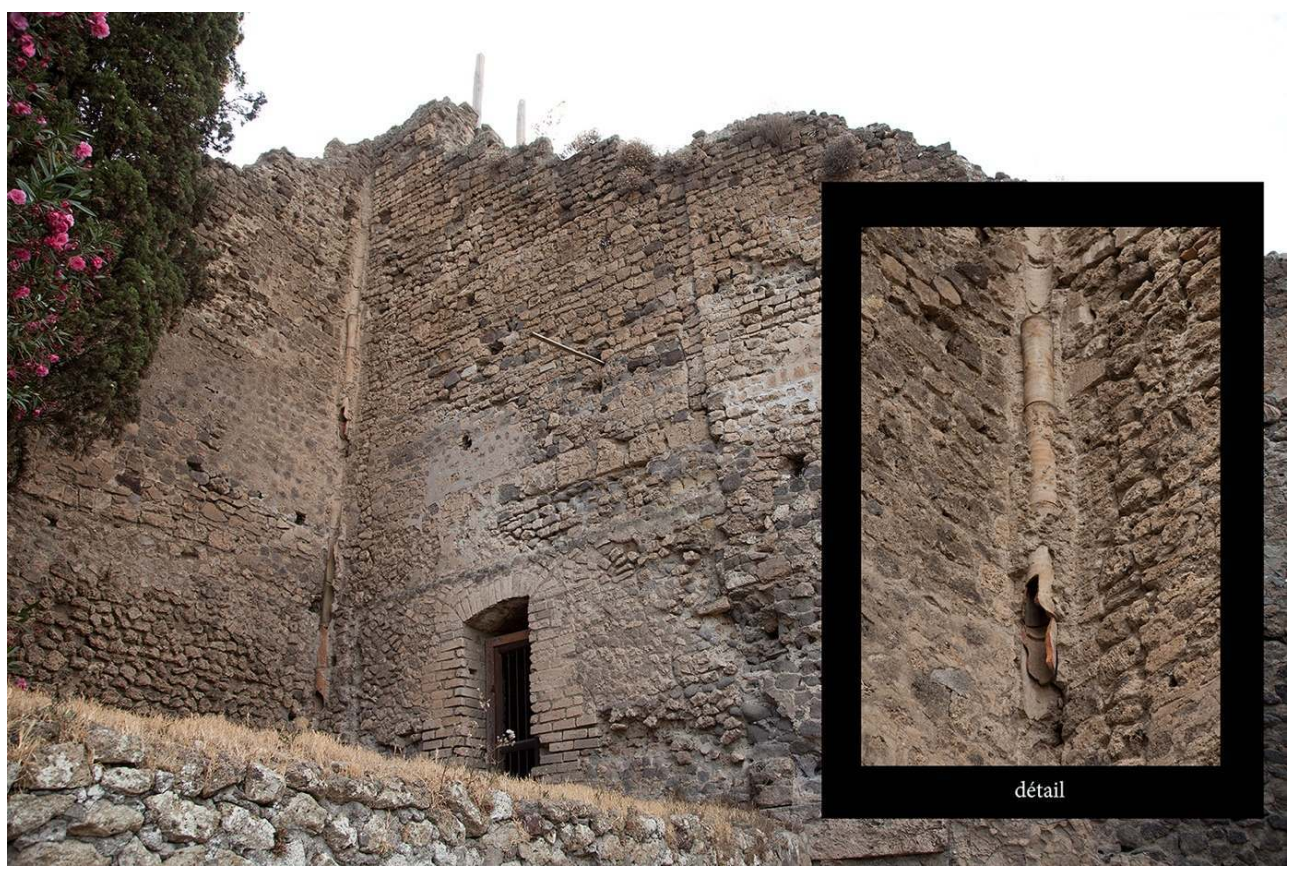

À l'arrière-plan on distingue les mâts remontés dans les consoles de fixation du velum.

Cl. Th. Crognier, avec l'autorisation du Parco Archeologico di Pompei, reproduction interdite.

Pour conclure, la partie haute de la summa cavea est bien une reconstruction entièrement moderne. Mais pour la disposition des consoles percées ainsi que pour celle des creusements destinées à laisser passer les mâts du velum dans les blocs de la corniche de couronnement en tuf, les restaurateurs du XVIII ${ }^{e}$ siècle se sont placés à l'aplomb des supports maçonnés que nous avons décrits et qui - pour les trois premiers d'entre eux au moins - sont bien antiques. La hauteur du dispositif et la disposition de la corniche n'étaient, quant à elles, peut-être pas assurées.

\section{Fiabilité de la restauration du dispositif de fixation du velum}

Il est donc nécessaire d'interroger la fiabilité de cette interprétation: le caractère unique de la disposition des consoles du velum à l'intérieur et non à l'extérieur de la summa cavea pourrait-il s'expliquer par une erreur de leur part? Pour répondre à cette question, on doit interroger les autres éléments-clés de la restitution du dispositif : les consoles percées en lave ${ }^{20}$ et la corniche en tuf gris de Nocera (ignimbrite) comportant des cavités pour le passage des mâts à l'aplomb des consoles (fig. 10). 
Fig. 10 - Corniche en tuf de la summa cavea présentant des cavités pour le passage des mâts du velum dans l'alignement des consoles percées en lave.

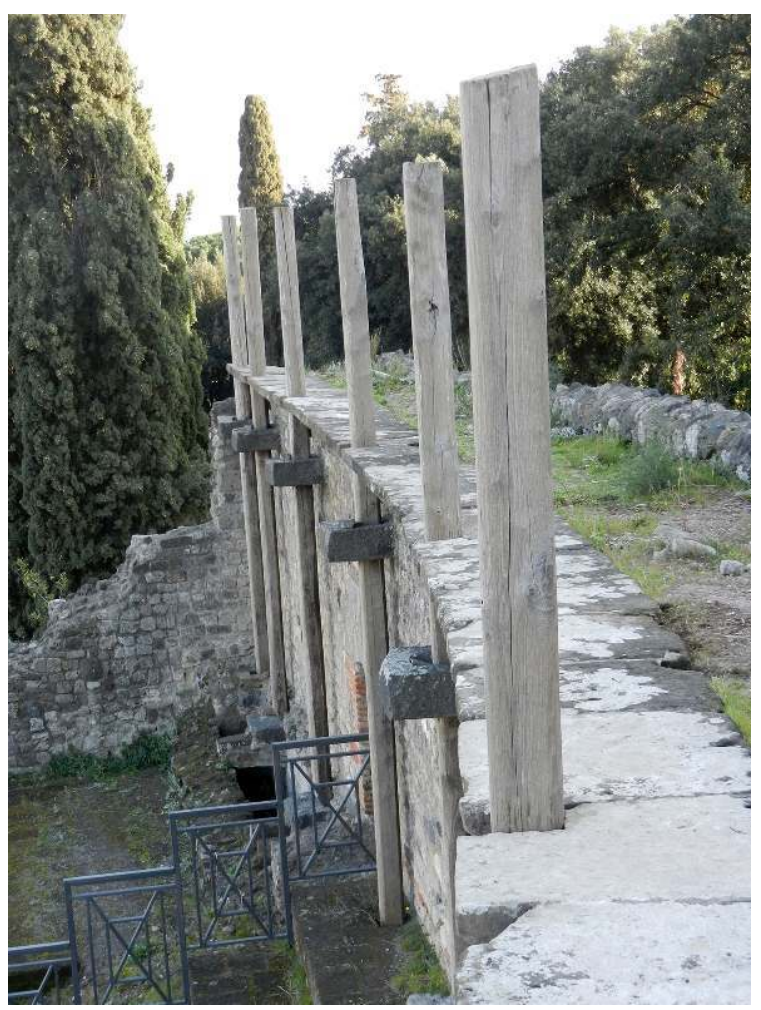

Cl. É. Letellier-Taillefer, avec l'autorisation du Parco Archeologico di Pompei, reproduction interdite.

15 Même si la découverte des consoles percées en lave n'est pas mentionnée dans les journaux de fouilles, il paraît peu probable que celles-ci soient le résultat d'une invention des tailleurs du pierre $d u$ XVIII ${ }^{\mathrm{e}}$ siècle. Il semble plus raisonnable de penser qu'elles ont été trouvées hors de leur position initiale pendant les dégagements. Reste à savoir si on n'en a trouvé que neuf - et dans ce cas pourquoi - ou bien si on a éliminé ou remployé ailleurs les autres consoles qui auraient été retrouvées mais n'auraient pas été nécessaires à la restauration seulement partielle de la summa cavea. Il est pour l'instant impossible de trancher sur ce point.

16 Notre observation des 52 blocs de la corniche en tuf ${ }^{21}$ a révélé que, si quelques-uns ont visiblement été remplacés très récemment ${ }^{22}$, tous les autres présentent des caractéristiques relativement homogènes malgré les variations de dimensions: utilisation du tuf gris de Nocera; nature et disposition des traces d'outils (grain d'orge et outil à tranchant plat); profil de mouluration comprenant un talon droit, souvent assez saillant et proportionnellement peu développé en hauteur, encadré de deux bandeaux; présence de cadres d'anathyrose sur les faces latérales lorsque celles-ci sont visibles; disposition de la cavité pour le mât toujours dans l'angle d'un bloc. Ces caractéristiques se retrouvent sur un bloc libre de corniche en tuf ${ }^{23}$ actuellement conservé au pied de la façade occidentale du theatrum tectum, qui incite à penser qu'il s'agit bien d'éléments antiques et non taillés ad hoc au XVIII ${ }^{\mathrm{e}}$ siècle $^{24}($ fig. 11, 12). 
Fig. 11 - Bloc en tuf de Nocera provenant de la corniche de la summa cavea du grand théâtre, actuellement conservé au pied de la façade occidentale du theatrum tectum.

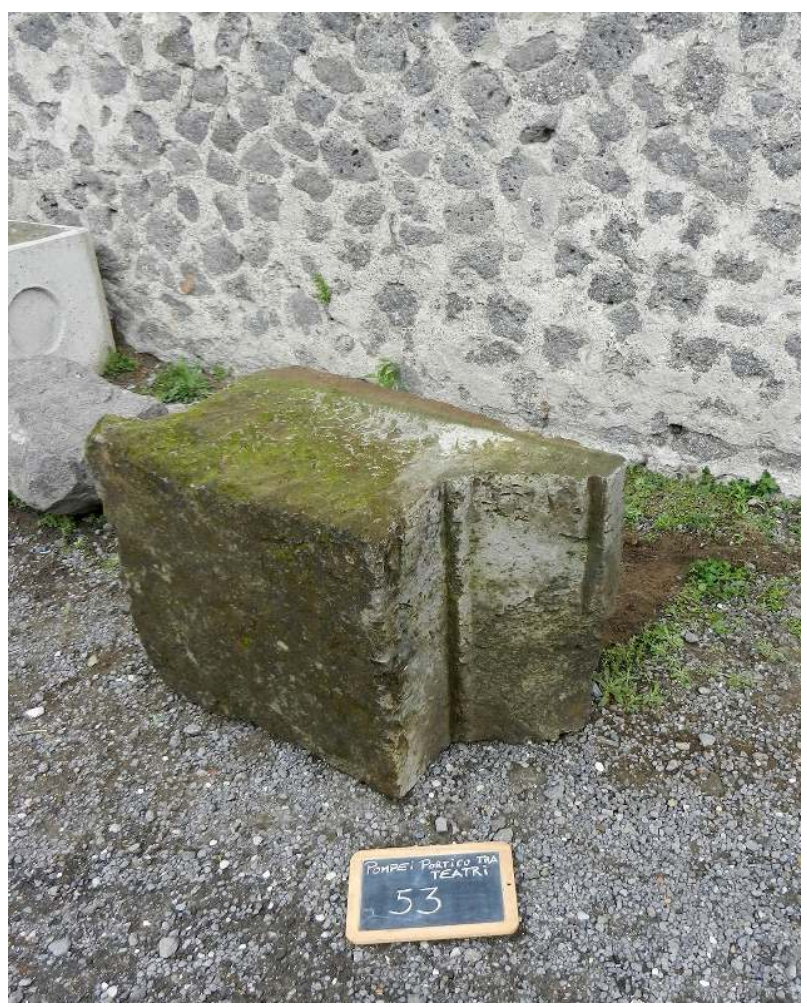

Cl. É. Letellier-Taillefer, avec l'autorisation du Parco Archeologico di Pompei, reproduction interdite

Fig. 12 - Relevé du bloc précédent.
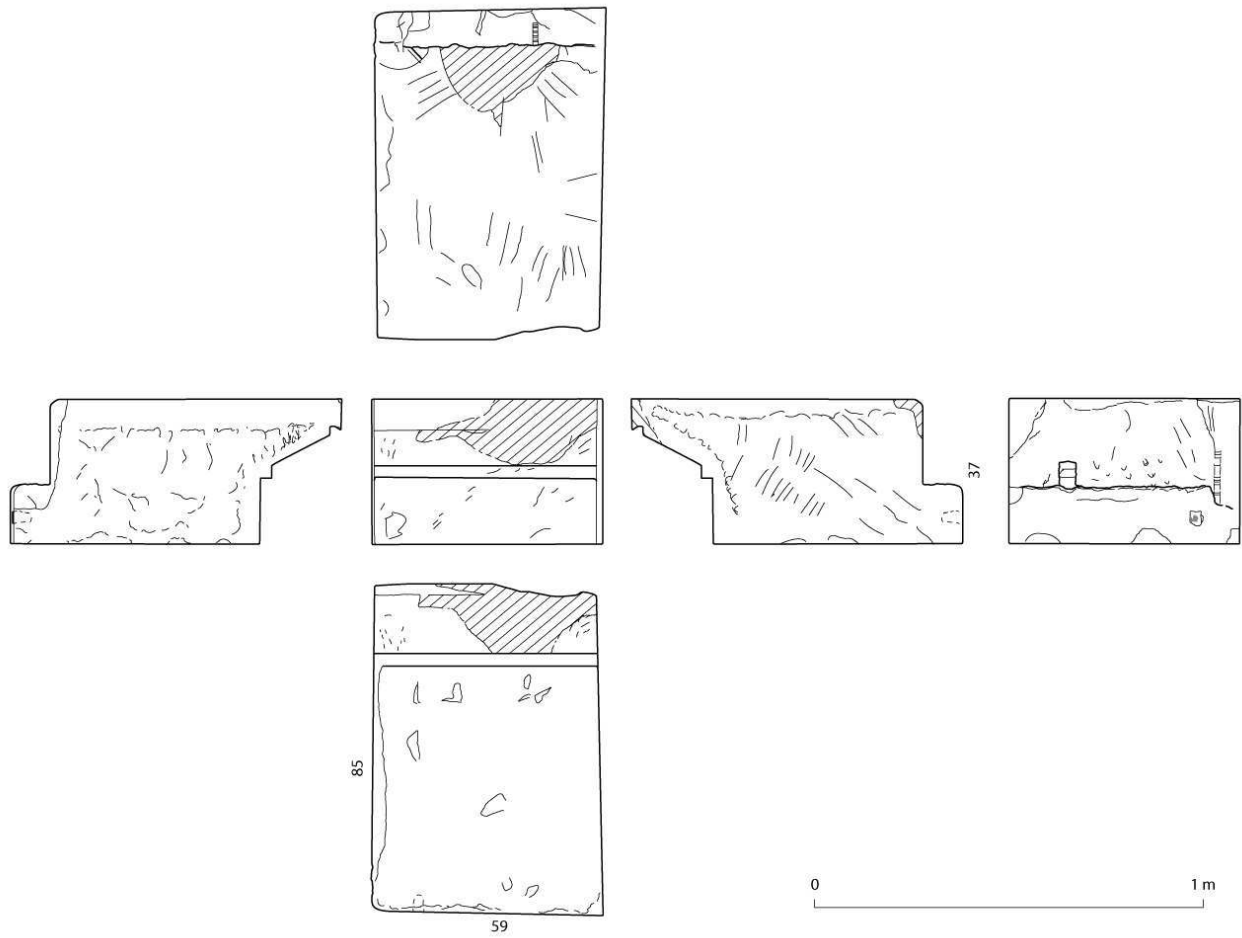
$1 \mathrm{~m}$

G. Chapelin, É. Letellier-Taillefer. 
17 Malgré cela, il demeure difficile de déterminer dans quelle mesure la disposition de ces blocs de corniche sur la courbe intérieure de la summa cavea peut être considérée comme fidèle à leur mise en œuvre antique. Certes, certains de ces blocs présentent une forme trapézoïdale plus ou moins marquée, qui pourrait confirmer leur provenance d'un élément courbe ${ }^{25}$. Cependant, ça n'est pas le cas de tous les blocs, et pour ceux qui présentent cette forme trapézoïdale, le côté le plus large est tantôt du côté de la face de parement ${ }^{26}$, tantôt du côté de la face arrière ${ }^{27}$, ce qui ne permet pas de restituer une courbe assurée. Enfin, la présence de mortaises de crampons situées à l'arrière du lit d'attente de plusieurs blocs de la corniche ${ }^{28}$ ou dans le creusement, observable seulement sur certains blocs, au niveau de l'arête entre lit d'attente et face arrière ${ }^{29}$, plaide pour une mise en œuvre initiale dans un contexte de grand appareil. On ne voit pas à quoi pouvaient servir ces crampons dans la situation actuelle, où les blocs sont insérés dans une construction maçonnée et couverts d'un sol en mortier de tuileau. Mais, comme c'est souvent le cas à Pompéi, un remploi de blocs de grand appareil n'est pas à exclure.

La corniche dans son état actuel reproduit donc une situation plausible même si elle n'est ni assurée ni sans doute entièrement fiable. On ne voit pas d'autre endroit où restituer ces éléments de décor. Quant à la disposition inhabituelle des mâts du velum à l'intérieur de la summa cavea, elle pourrait aussi s'expliquer non par une erreur des restaurateurs mais par la disposition particulière du théâtre de Pompéi, mitoyen de l'aile orientale $d u$ forum triangulaire: l'absence d'une courbe extérieure libre de ce côté de la cavea empêchait-elle d'y fixer les consoles? Dans cette configuration avec les consoles à l'intérieur, la traction exercée en haut du mât par le velum devait engendrer un effort de traction considérable sur les consoles. La résistance à la traction n'est pas la qualité première des pierres. Un facteur limitant a donc dû être la solidité des parties de lave les plus sollicitées, celles proches du percement des consoles.

\section{Velum et dispositif hydraulique?}

Un dernier problème archéologique se pose à propos des traces de ce dispositif de fixation du velum. On observe qu'au niveau des cavités maçonnées interprétées comme des supports pour les mâts, les parties antiques du mur périphérique de la summa cavea, en appareil irrégulier de calcaire, présentent une bande verticale en fragments de terres cuites architecturales dans l'alignement $d u$ mât ${ }^{30}$. Cette particularité constructive pourrait avoir servi à renforcer et régulariser le mur là où le mât venait s'appuyer (fig. 13). 
Fig. 13 - Deuxième support maçonné au-dessus de la structure creuse, la ligne de terre cuite est visible derrière le mât en bois.

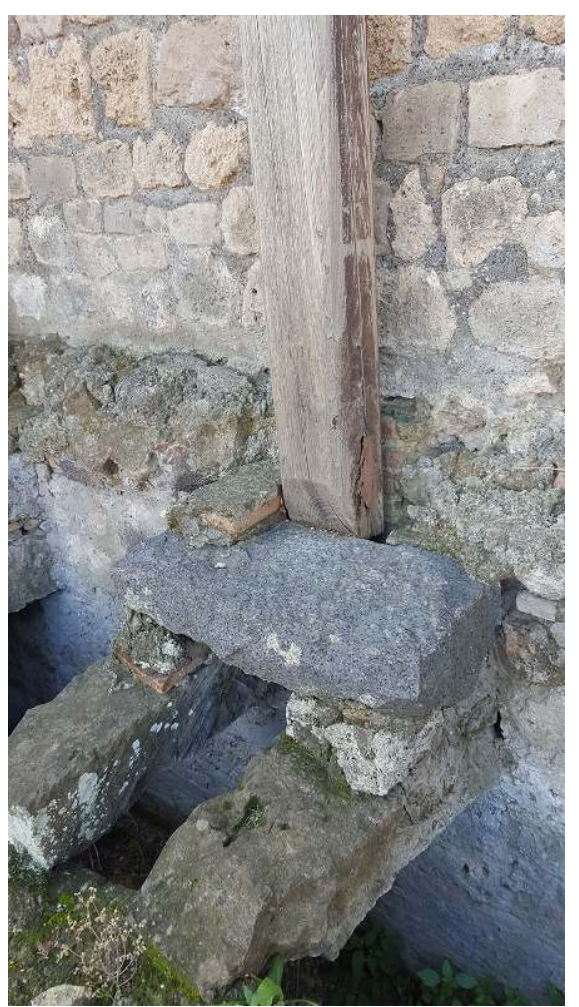

Cl. É. Letellier-Taillefer, avec l'autorisation du Parco Archeologico di Pompei, reproduction interdite.

20 Les bandes verticales de terres cuites, visibles avant les travaux de 2010 dans l'alignement de toutes les consoles de mâts (fig. 14), étaient probablement issues, pour une partie d'entre elles, des restaurations du xvIII ${ }^{e}$ siècle, sur les modèles des trois premières. 
Fig. 14 - Crypta et summa cavea du grand théâtre avant les travaux de 2010.

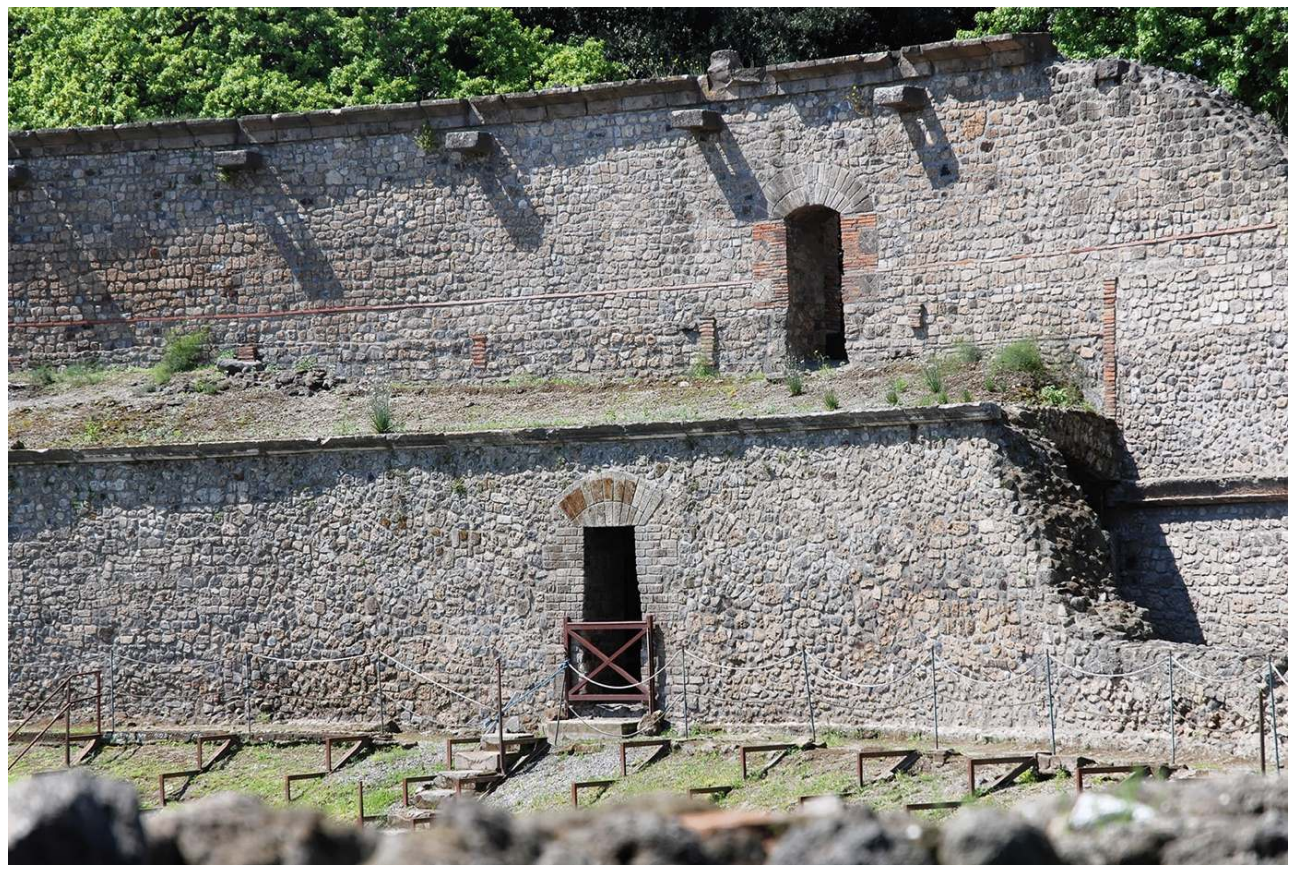

Cl. É. Letellier-Taillefer, avec l'autorisation du Parco Archeologico di Pompei, reproduction interdite.

Dans l'alignement des deux derniers mâts conservés ${ }^{31}$ (fig. 14), les bandes de terres cuites se prolongent à travers la voûte de la crypta et interrompent son imposte en terre cuite à l'intérieur du couloir (fig. 15). Ces deux bandes «traversantes ", même si elles sont peut-être aussi en partie restaurées, ne l'ont pas été au hasard ${ }^{32}$, mais certainement sur la foi d'indices antiques. Nous notons qu'elles apparaissent précisément dans l'alignement du départ de la rigole maçonnée couverte de mortier hydraulique qui court au pied du mur périphérique de la crypta. Ces bandes nous semblent dès lors s'apparenter à des descentes d'eau. On imagine mal pourquoi les restaurateurs auraient « inventé » ce système traversant peu intuitif et on pressent une fonction hydraulique, liée à la structure creuse de la summa cavea.

Par ailleurs, on observe aussi deux autres descentes d'eau maçonnées en terre cuite qui débouchent, cette fois, à l'extérieur du mur périphérique de la crypta, dans la paroi en appareil irrégulier de lave et calcaire des latrines ménagées entre la courbe de la cavea du théâtre et le mur du forum triangulaire ${ }^{33}$. Il y a donc, là encore, un lien entre les structures de la summa cavea et des aménagements liés à l'eau aux étages inférieurs. 
Fig. 15 - Descente d'eau en terre cuite provenant de l'étage supérieur au niveau du départ de la rigole maçonnée de la crypta, dans l'alignement du mât $n$. 9.

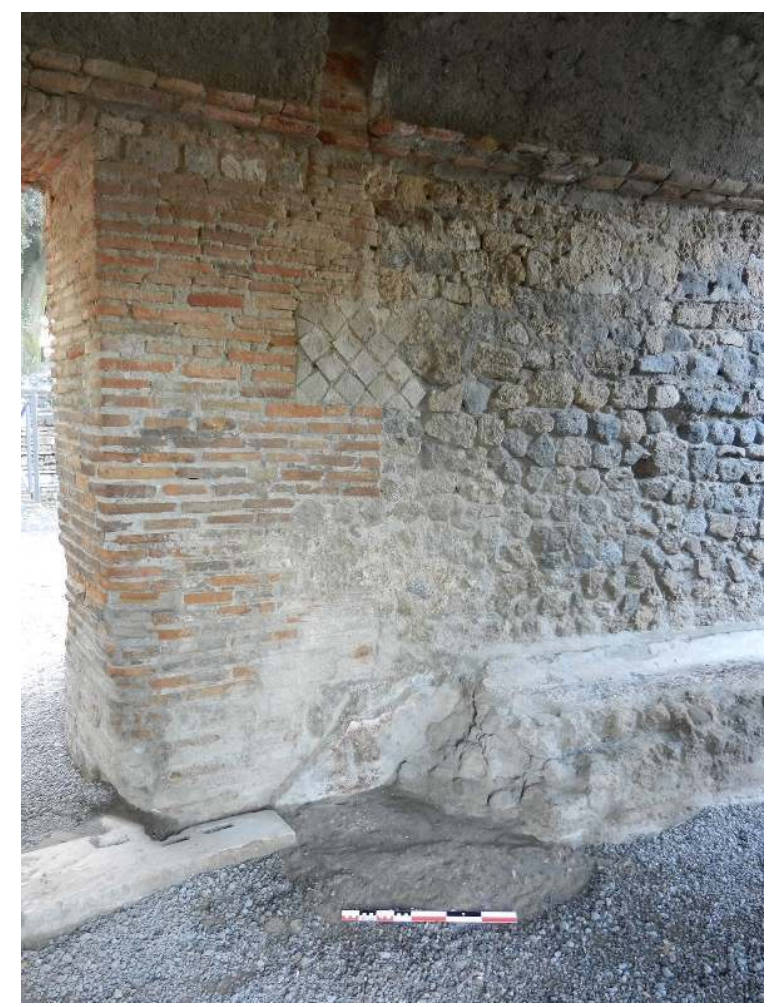

Cl. É. Letellier-Taillefer, avec l'autorisation du Parco Archeologico di Pompei, reproduction interdite.

La fonction précise de ce système (évacuation des ruissellements destinée à protéger les mâts du velum des risques de pourrissement?) sera interrogée lors de prochaines campagnes du projet Teatri di Pompei, que nous souhaitons consacrer au système hydraulique des théâtres. Sa compréhension nous permettra alors peut-être d'affiner notre analyse de la structure creuse de la summa cavea (simple vide destiné à alléger le bâti ou dispositif hydraulique ?) et, par la même occasion, de mieux comprendre comment restituer le dispositif du velum.

\section{Étude des graffitis du couloir VIII 7, 20}

24 La deuxième campagne de terrain de l'année 2017 a permis d'entamer le relevé graphique et photographique des graffitis qui couvrent les enduits des deux parois du couloir d'accès commun aux deux théâtres (VIII 7, 20) (fig. 16). 
Fig. 16 - Le couloir des théâtres de Pompéi (VIII 7, 20), vue générale depuis la rue de Stabies.

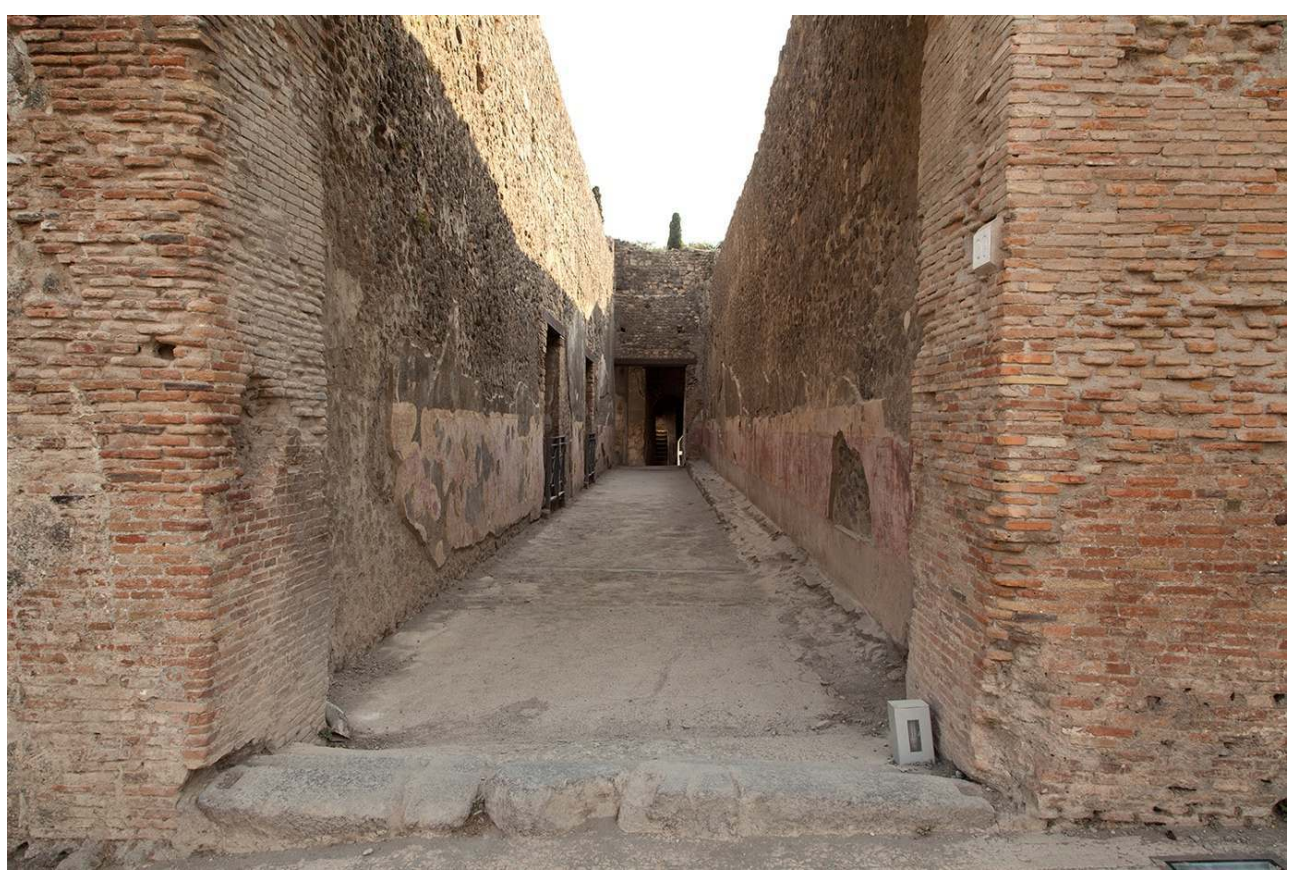

Cl. Th. Crognier - EFR/IRAA, avec l'autorisation du Parco Archeologico di Pompei, reproduction interdite.

Cet espace aménagé au moment de la construction du theatrum tectum, desservait les deux théâtres depuis la rue de Stabies. Au-delà des deux portes permettant d'emprunter les escaliers d'accès au sommet de la cavea du theatrum tectum, le couloir, qui pouvait être clos, débouche dans l'aditus oriental du grand théâtre. Ses murs étaient couverts d'enduit peint, relativement bien conservé en partie basse, où l'on observe un registre inférieur rouge surmonté d'une bande jaune. Le registre supérieur a presque entièrement disparu. Sur l'ensemble des parties conservées de l'enduit (jusqu'à 1,50 m de hauteur, sur les $27 \mathrm{~m}$ de longueur du couloir), on distingue encore de nombreux graffitis, textuels comme figurés. Même si l'érosion progressive de l'enduit rend leur lecture parfois difficile aujourd'hui, la plupart des textes et des images avaient précédemment été lus et recensés un par $u^{34}$ et quelques études thématiques en ont découlé35.

Les plus anciens graffitis de ce couloir datent de la fin de la République, comme l'indique notamment une date consulaire remontant à 37 av. J.-C. ${ }^{36}$ Certains ont été partiellement recouverts à l'occasion de l'installation, au pied de la paroi nord, d'une rigole maçonnée couverte de mortier hydraulique. Celle-ci fut peut-être aménagée au moment du raccordement des théâtres à un réseau d'eau sous pression, à l'époque augustéenne; nous sommes enclins à y reconnaître un urinoir, équipement incontournable dans des lieux de rassemblement tels que les théâtres. Même si la grande majorité des graffitis sont impossibles à dater précisément, il est probable qu'une partie d'entre eux soient postérieurs à cet aménagement : certains évoquent d'ailleurs la fonction d'urinoir ${ }^{37}$ (fig. 17). Les plus récents remontent sans doute aux dernières années avant l'éruption, le grand théâtre étant alors toujours en fonction ${ }^{38}$. L'ampleur de la période chronologique concernée se traduit dans l'accumulation et la superposition des tracés, qui dénote également la fréquentation du lieu. 
Fig. 17 - Relevé manuel de graffitis dont l'un représente un personnage urinant (mur nord du couloir VIII 7, 20).

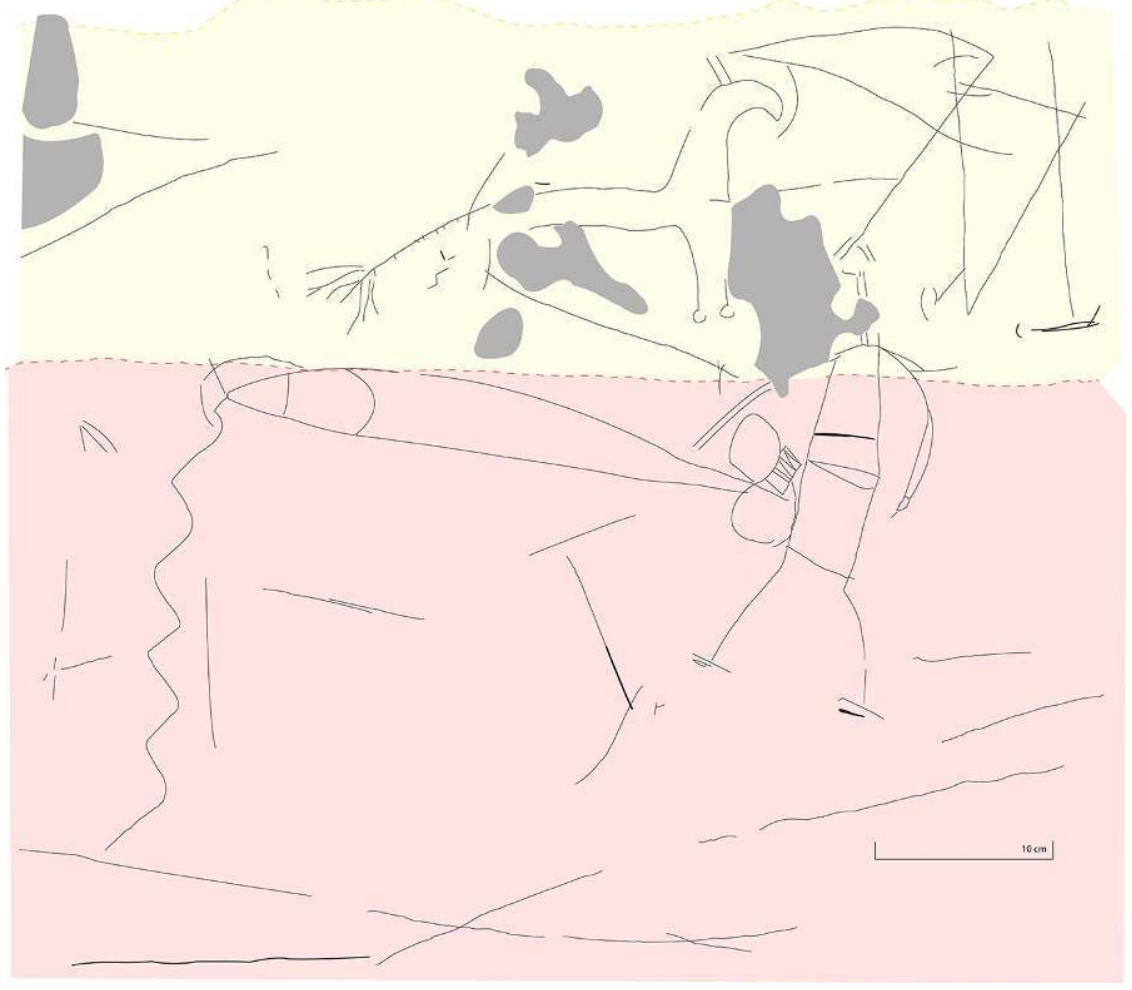

Relevé J. Hiault-Echeverria, DAO É. Letellier-Taillefer.

Aussi s'agit-il d'un ensemble d'inscriptions exceptionnel qui matérialise la vivacité de la vie quotidienne et des échanges qui avaient lieu dans cet espace non seulement d'accès, mais aussi sans doute d'attente, de passage, de déambulation ou, peut-être, de séjour prolongé. C'est pourquoi il nous a semblé nécessaire de l'aborder non plus seulement comme une collection de mots et d'images de types variés mais comme un ensemble signifiant et complexe, dont nous entendons explorer le fonctionnement global.

C'est dans ce but que nous avons entrepris un relevé graphique et photographique complet des deux parois. La couverture photographique a été réalisée par Th. Crognier et associée à un relevé topographique de cibles qui permettent d'utiliser les clichés pour élaborer un modèle photogrammétrique des parois sur lequel nous pouvons situer chacun des graffitis et observer leurs relations (fig. 18). 
Fig. 18 - Montage des orthophotographies des murs nord (en haut) et sud (en bas) du couloir VII 7, 20 et détails de quelques graffitis en prises de vue RTI.

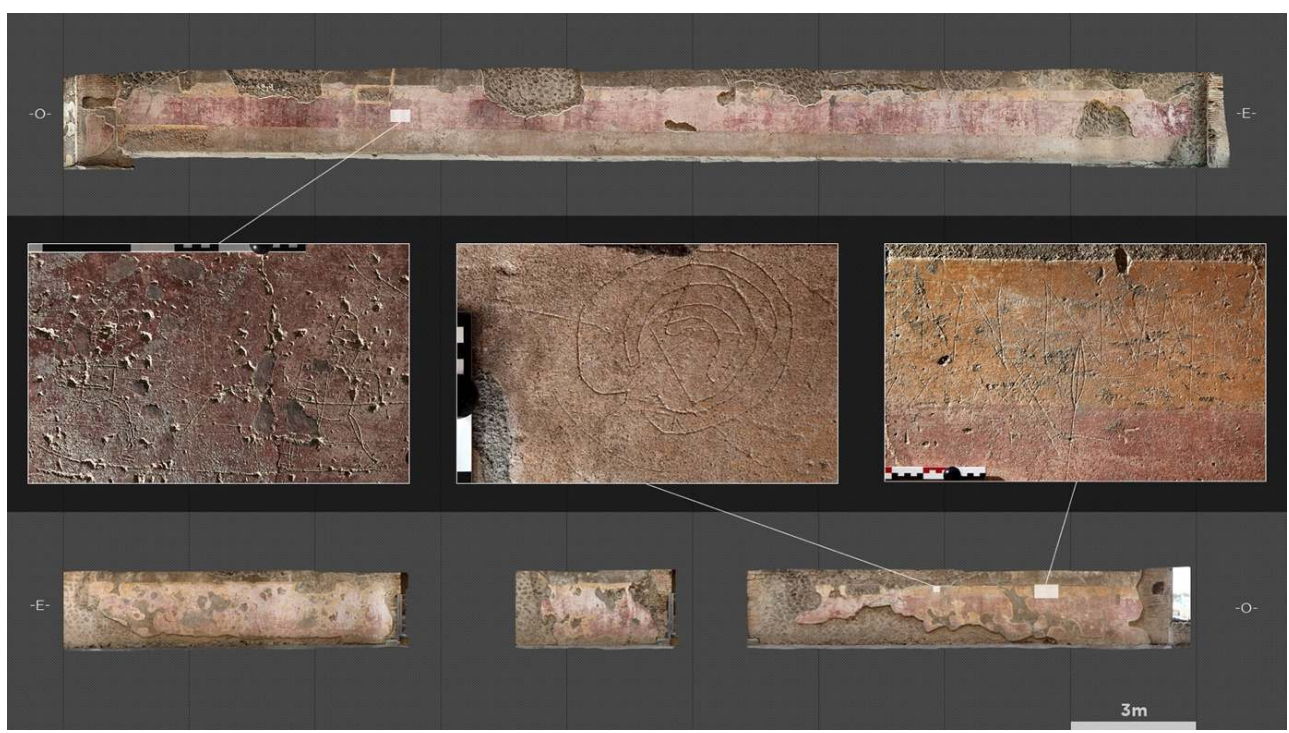

Cl. et montage Th. Crognier - EFR/IRAA.

Le relevé graphique manuel, effectué au calque, n'a pas pu être exhaustif dans le temps imparti mais une grande majorité des graffitis a tout de même été couverte. Cette approche physique directe nous a notamment permis de repérer différents types d'outils utilisés, plus ou moins pointus ou tranchants. Le relevé manuel nous a également amenés à faire l'expérience des gestes d'écriture sur le mur, ce qui nous a par exemple conduits à émettre l'hypothèse de possibles séjours prolongés dans ce couloir, puisque nous avons pu constater que certains dessins ont nécessairement été réalisés en position assise au sol.

Enfin, pour quelques ensembles particulièrement complexes ou difficiles à lire, nous avons appliqué la technique de prises de vues RTI (Reflectance Transformation Imaging ${ }^{39}$ qui facilite la lecture des graffitis en recomposant un modèle numérique de l'inscription au sein duquel il est possible de faire varier l'orientation d'une source de lumière rasante (cf. fig. 19). Cet outil permet de mieux lire les détails des micro-reliefs de l'incision et peut compléter l'observation à l'œil nu. La technique n'est cependant applicable qu'à de petites surfaces. 
Fig. 19 - Exemple de rendus du modèle RTI pour un graffiti représentant un labyrinthe sur le mur sud du couloir.

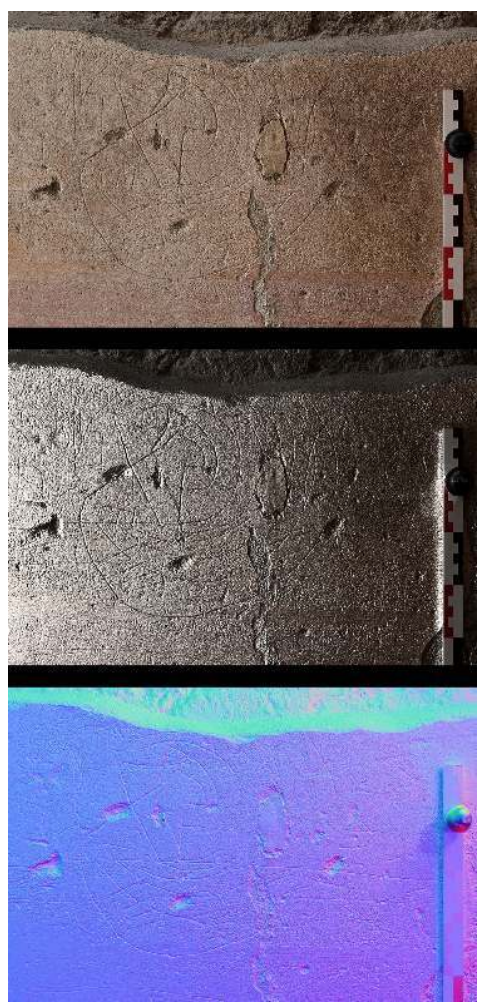

Le détail d'un portrait de profil, mal lisible à l'œil nu, apparaît à côté du labyrinthe, en haut à gauche, sur les deux dernières images.

$\mathrm{Cl}$. et montage RTI Th. Crognier.

En raison d'une interruption temporaire de l'activité du projet Teatri di Pompei durant l'année 2018, le traitement des données rassemblées est encore en cours. Certains ensembles de graffitis ont d'ores et déjà pu être analysés dans le cadre d'enquêtes thématiques, par exemple sur les jeux graphiques et de références visuelles autour de schémas de labyrinthes ${ }^{40}$ ou encore sur l'usage possible de ce couloir comme un lieu de séjour et d'accuei ${ }^{41}$. Nous poursuivons ces recherches dans le cadre d'une réflexion sur la pluralité des discours qui pouvaient se déployer dans les édifices de spectacles, sur scène et dans les gradins, mais aussi dans les inscriptions officielles affichées sur les monuments comme dans les graffitis qui y furent plus furtivement incisés. Il s'agit d'une des voies de compréhension du fonctionnement de ces édifices comme des lieux publics. 


\section{BIBLIOGRAPHIE}

Boulenger 1603 = J.C. Boulenger, De theatro ludisque scenicis libri duo, Troyes, Chevillot, 1603.

Capelle 2017 = J. Capelle, Reflectance Transformation Imaging (RTI) et épigraphie, dans RAAN.

Recherche en architecture, archéologie et numérique - un blog de l'IRAA, publié le 20/02/2017 : https:// raan.hypotheses.org/1326

Castrén 1983 = P. Castrén, Ordo populusque Pompeianus: polity and society in Roman Pompeii, Roma, 1983.

Della Corte 1924 = M. Della Corte, Iuventus: Un nuovo aspetto della vita pubblica di Pompei finora inesplorato, studiato e ricostruito con la scorta dei relativi documenti epigrafici, topografici, demografici, artistici e religiosi, Arpino, 1924.

Dessales et al. 2016 = H. Dessales, J. Ponce, C. Boust, G. Chapelin, M. Carrive, J. Cavero, A. Coutelas, R. Deiana, M. Di Ludovico, G. De Martino, J. Dubouloz, É. Letellier-Taillefer, A. Maigret,

G. Manfredi, F. Marchand-Beaulieu, A. Milanese, C. Modena, F. Monier, A. d'Harcourt-Péron, A.B. Pimpaud, A. Prota, E. Rizzo, A. Rossi, A. Santoriello, A. Tricoche, M.R. Valluzzi, Pompéi. Villa de Diomède. Campagne d'étude 2015, dans Chronique des activités archéologiques de l'École française de Rome, 2016 : https://journals.openedition.org/cefr/1543

DiBiasie Sammons 2018 = J.F. DiBiasie Sammons, Application of Reflectance Transformation Imaging (RTI) to the study of ancient graffiti from Herculaneum, Italy, dans Journal of Archaeological Science: Reports, 17, Supplement C, 2018, p. 184-194.

Fincker - Letellier-Taillefer - Zugmeyer 2018 = M. Fincker, É. Letellier-Taillefer, S. Zugmeyer, Théâtres de Pompéi - Campagne 2016, dans Chronique des activités archéologiques de l'École française de Rome, 2018 : https://journals.openedition.org/cefr/1853

Frood - Howley 2014 = E. Frood, K. Howley, application of reflectance transformation imaging (rti) in the study of Temple Graffiti, dans E. Pischikova, J. Budka, K. Griffin (dir.), Thebes in the first millenium BC, Newcastle upon Tyne, 2014, p. 625-638.

Galiani 1790 = B. Galiani, L'architettura di Marco Vitruvio Pollione, Napoli, Fratelli Terres, 1790, 2éd.

García y García 2006 = L. García y García, Danni di guerra a Pompei: una dolorosa vicenda quasi dimenticata, con numerose notizie sul «Museo pompeiano» distrutto nel 1943, Roma, 2006 (Studi della Soprintendenza archeologica di Pompei, 15).

Graefe 1979 = R. Graefe, Vela erunt: die Zeltdächer der römischen Theater und ähnlicher Anlagen, Mainz am Rhein, 1979.

Isler 2017 = H.P. Isler, Antike Theaterbauten: ein Handbuch, Vienne, 2017.

Keegan 2011 = P. Keegan, Blogging Rome. Graffiti as speech-act and cultural discourse, dans J.A. Baird, C. Taylor (dir.), Ancient graffiti in context, New York, 2011, p. 165-190.

Langner 2001 = M. Langner, Antike Graffitizeichnungen: Motive, Gestaltung und Bedeutung, Wiesbaden, 2001 (Palilia, 11).

Le Gall 1954 = J. Le Gall, Graffites navals du Palatin et de Pompéi, dans Mémoires de la Société Nationale des Antiquaires de France, ser. 9, t. 3, 1954, p. 41-52. 
Letellier-Taillefer 2018 = É. Letellier-Taillefer, Textes, images et imaginaire de l'architecture: dans le dédale des graffitis labyrinthiques du couloir des théâtres de Pompéi, dans R. Robert, G. Viard (dir.), Les architectures fictives de l'Antiquité : textes - images, Bordeaux, 2018, p. 179-209.

Letellier-Taillefer 2019 = É. Letellier-Taillefer, Nouvelles recherches sur les théâtres de Pompéi, dans Revue archéologique, 2019, 1, p. 178-184.

Letellier-Taillefer à paraître = É. Letellier-Taillefer, Les édifices de spectacles romains : lieux publics, lieux d'accueil, lieux d'hospitalité ?, dans Topoi, à paraître.

Maiuri 1958 = A. Maiuri, Navalia Pompeiana, dans Rendiconti della Accademia di archeologia lettere e belle arti di Napoli, n.s. 33, 1958, p. 7-34.

Mau 1908 = A. Mau, Pompeji in Leben und Kunst: mit einem Kapitel über Herculaneum, Zweite verbesserte und vermehrte Auflage mit einem Kapitelüber Herculaneum, Leipzig, 1908.

Navarre s.d. = O. Navarre, Velum et Velarium, dans C. Daremberg, E. Saglio (éd.), Dictionnaire des antiquités grecques et romaines, Paris, Hachette, s.d., p. 677-680.

Nissen 1877 = H. Nissen, Pompeianische Studien zur Städtekunde des Altertums, Leipzig, 1877.

Overbeck - Mau 1884 = J. Overbeck, A. Mau, Pompeji in seinen Gebäuden, Altertümern und Kunstwerken, Leipzig, 1884.

Patte $1782=$ M. Patte, Essai sur l'architecture théâtrale, ou, De l'ordonnance la plus avantageuse à une salle de spectacles, relativement aux principes de l'optique \& de l'acoustique : avec un examen des principaux théâtres de l'Europe, \& une analyse des écrits les plus importans sur cette matiere, Paris, Moutard, 1782.

Saunders $1790=\mathrm{G}$. Saunders, A treatise on theatres, London, Printed for the author and sold by I. and J. Taylor, at the Architectural library, Holborn, 1790.

Varone 1994 = A. Varone, Erotica pompeiana: iscrizioni d'amore sui muri di Pompei, Roma, 1994 (Studia archaeologica, 71).

Winckelmann 1764 = J.J. Winckelmann, Nachrichten von den neuesten Herculanischen Entdeckungen [édition 1997], Mainz am Rhein, 1764 (Schriften und Nachlass, 2,2).

\section{NOTES}

1. Présentation du projet et premiers résultats dans Fincker - Letellier-Taillefer Zugmeyer 2018 ; Letellier-Taillefer 2019.

2. Ces documents ont fait l'objet de recherches approfondies dont les résultats ont été exposés dans un mémoire de recherche de l'École française de Rome présenté par É. Letellier-Taillefer à l'Académie des inscriptions et belles-lettres en juin 2017. Certains sont présentés ici, l'ensemble fera l'objet d'une publication dans le cadre du projet Teatri di Pompei.

3. Voir Graefe 1979, p. 36-40 et pl. 28-35, où l'auteur avait bien repéré que la summa cavea était largement restaurée mais manquait d'informations sur ces restaurations; cf. également Isler 2017, vol. 1, p. 762.

4. R. Graefe cite pour seule exception, et seulement partielle, l'amphithéâtre de Cassino: Graefe 1979, p. 40.

5. Cf. García y García 2006, fig. 339, p. 141.

6. Voir les rapports d'intervention du $1^{\text {er }}$ février 1944 au $1^{\text {er }}$ décembre 1946 dans les archives de la Surintendance. 
7. Les photographies des spectacles donnés dans le théâtre dans les années 1950 montrent que la crypta n'était alors pas encore entièrement remontée.

8. La position d'O. Navarre dans son article sur le velum pour le Dictionnaire des Antiquités grecques et romaines était très sceptique : Navarre s.d., p. 677 ; l'affirmation plus récente de H.-P. Isler, qui estime d'emblée que la restauration des consoles à l'intérieur de la cavea est erronée (Isler 2017, vol. 1 , note 8047 , p. 758 et vol. 2, p. 626) nous semble trop rapide.

9. Cf. Overbeck - Mau 1884, p. 164.

10. De Architectura, X, praef. 3-4.

11. Par exemple: Boulenger 1603, p. 33 ; Patte 1782, p. 47 ; Saunders 1790, p. 49 ; évoquant le velum dans une note de sa traduction de Vitruve, Berardo Galiani, qui disposait d'informations de première main sur les fouilles d'Herculanum et Pompéi, ne mentionne pas d'attestations archéologiques, même dans sa seconde édition : Galiani 1790, note 1, p. 223.

12. Winckelmann 1764, p. 17 (1. 10-11)

13. Dans son ouvrage spécifiquement consacré à la question du velum $\mathrm{R}$. Graefe mentionne seulement que les plus anciennes restitutions du système datent du Xvi s. : Graefe 1979, p. 1.

14. Par convention, nous décrivons l'édifice comme si son axe de symétrie était parfaitement orienté nord-sud.

15. Voir Fincker - Letellier-Taillefer - Zugmeyer 2018, fig. 1.

16. Le seul à l'avoir précisément décrite, sans pouvoir déterminer sa fonction, est R. Graefe : Graefe 1979, p. 38-39.

17. Du sol actuel jusqu'au départ de la voûte.

18. Graefe 1979, p. 38. Le mortier visible actuellement est-il issu d'une restauration moderne postérieure à 1979, éventuellement des restaurations de 2010 ? Ce point pourrait être clarifié par une investigation dans la partie actuellement comblée de déblais sous les gradins restaurés en 2010.

19. Voir Fincker - Letellier-Taillefer - Zugmeyer 2018, notamment fig. 6, pour une analyse de la façade sud du théâtre.

20. Dimensions moyennes de la partie visible $=53 \times 42 \times 17 \mathrm{~cm}$. Dimensions moyennes de la cavité pour le mât : $17 \times 25 \mathrm{~cm}$.

21. Nous les avons recensés et numérotés ( $\mathrm{C} 1$ à $\mathrm{C} 52)$ en commençant par l'extrémité sud.

22. Notamment les blocs $C 12,13,14$ et 16 : certainement à l'occasion des restaurations des années 1950 destinées à réparer les dégâts causés par le bombardement de 1943.

23. Nous l'avons numéroté $C 53$.

24. Ce que la permanence des techniques et outils de taille de pierre depuis l'Antiquité jusqu'à cette époque aurait rendu difficile à distinguer. Peut-être les «blocs de corniche en pierre tendre » signalés en septembre 1764, alors qu'on découvrait les parties hautes du théâtre font-ils partie de cette série, à moins qu'il ne s'agisse plutôt de la corniche du portique du forum triangulaire. (Rapport du 22 septembre 1764 : « due pezzi di cornice di piperno dolce »)

25. Comme l'avait suggéré l'excellent connaisseur de ce théâtre August Mau : Mau 1908, p. 144.

26. $\mathrm{C} 6, \mathrm{C} 11$.

27. $\mathrm{C} 26, \mathrm{C} 28, \mathrm{C} 30, \mathrm{C} 39$.

28. $\mathrm{C} 27,29,30,32,34,35,37,49,50$.

29. $\mathrm{C} 1, \mathrm{C} 53$.

30. Observable aujourd'hui pour les trois premiers supports à partir de l'extrémité sud-ouest de la cavea.

31. La dixième console percée en lave s'est brisée, la partie manquante est actuellement conservée au pied des gradins de la summa cavea.

32. R. Graefe expliquait la différence de profondeur entre ces bandes de terre cuite par l'« incertitude » des restaurateurs : Graefe 1979, p. 39. Mais au vu de notre analyse des pratiques de restaurations de Fr. La Vega, l'argument ne parait pas suffisant pour expliquer cette variation. 
33. L'éventuel alignement de ces deux descentes d'eau avec les structures du velum correspondantes, dans cette partie centrale, très restaurée, doit encore faire l'objet de vérifications par relevé topographique.

34. CIL X, 2414-2457 pour les textes, et Langner 2001 pour les images.

35. Le Gall 1954 ; Maiuri 1958, p. 28-32 ; Varone 1994 ; Keegan 2011, p. 183.

36. CIL IV, 2437. Cf. Nissen 1877, p. 240 ; Della Corte 1924, p. 33 ; Castrén 1983, note 2, p. 96.

37. CIL IV, 2416 : MICCIO COCIO [..]V TVO PATRI CACANTI CONFREGISTI PIIRAM / MICCIONIS STATVM COSIDERATE et le dessin recensé sous le numéro 1263 par M. Langner (cf. fig. 17).

38. Cf. Letellier-Taillefer 2019.

39. Cette technique se développe depuis plusieurs années dans le domaine de l'archéologie et en particulier pour l'analyse des inscriptions et des graffitis historiques, par exemple: Frood Howley 2014 ; Dessales et al. 2016 ; Capelle 2017 ; DiBiasie Sammons 2018.

40. Letellier-Taillefer 2018.

41. Letellier-Taillefer à paraître.

INDEX

institutions École française de Rome - Centre Jean Bérard, Institut de Recherche sur l'Architecture Antique (USR 3155 CNRS-AMU), Parco Archeologico di Pompei

Index géographique : Pompéi

Mots-clés : Pompéi, architecture, théâtre, velum, graffiti

\section{AUTEURS}

\section{ÉLOÏSE LETELLIER-TAILLEFER}

Sorbonne Université

\section{GUILHEM CHAPELIN}

Centre Jean Bérard, USR 3133 CNRS-EFR 